\title{
12.1
}

\section{Исследование состава остаточного газа в вакуумной системе циклотрона ФТИ им. А.Ф. Иоффе}

\author{
(ㄱ Н.Н. Аруев, М.А. Козловский, М.Ф. Кудояров , М.Я. Патрова, П.А. Романов, Р.В. Тюкальцев, \\ И.Л. Федичкин, С.В. Филиппов
}

Физико-технический институт им. А.Ф. Иофффе РАН, Санкт-Петербург, Россия

๑ E-mail: mkud@cycla.ioffe.ru

Поступило в Редакцию 29 апреля 2019г.

В окончательной редакции 13 мая 2019г

Принято к публикации 13 мая 2019 г.

\begin{abstract}
С помощью разработанного в ФТИ малогабаритного времяпролетного масс-спектрометра ФТ-200 получены спектры масс молекул остаточного газа в вакуумной системе циклотрона ФТИ им. А.Ф. Иоффе в различных условиях эксперимента. Измерения проводились в вакуумной камере циклотрона и в оконечной части ионопровода вблизи камеры облучения образцов. Установлено, что основной вклад в состав остаточного газа в камере циклотрона вносят молекулы используемого вакуумного масла (до 82 vol.\%) и молекулы воды (до $15-17 \mathrm{vol} . \%)$, а в ионопроводе вблизи камеры облучения с полимерной пленкой молекулы воды (до 63 vol.\%). Полученные данные являются основанием для модернизации циклотронного комплекса ФТИ.
\end{abstract}

Ключевые слова: времяпролетный масс-спектрометр, вакуумная система циклотрона, состав остаточного газа.

DOI: 10.21883/PJTF.2019.16.48153.17860

Изучение спектров масс молекул остаточного газа в вакуумных системах крупных ядерно-физических установок играет весьма важную роль, так как дает возможность понять, детально исследовать и воздействовать на процессы, происходящие при работе этих сложнейших инструментов, путем введения новых и модернизации старых элементов в конструкции установки.

В качестве примера, подтверждающего важность указанных исследований, можно сослаться на работы [1-3], выполненные сотрудниками ОИЯИ. Результаты этих работ послужили основанием для глубокой модернизации существовавших ускорителей тяжелых ионов У-200, МЦ-400, ИЦ-100, а также разработки и изготовления новых ускорителей ДЦ-60 и ДЦ-110. В первую очередь модернизация привела к замене внутренних источников ионов типа Пеннинга (PIG-источники) на внешние ионные источники, основанные на эффекте электронноциклотронного резонанса (ЭЦР-источники), что кардинально изменило ситуацию с вакуумом внутри камеры циклотрона и привело к увеличению эффективности вывода из циклотрона тяжелых многозарядных ионов.

Заметим, что ЭЦР-источники используются для получения тяжелых ионов, в то время как в уникальном циклотроне ФТИ им. А.Ф. Иоффе используется внутренний PIG-источник, который дает возможность получать пучки ускоренных ионов средних масс (до $m=40 \mathrm{u}$ ), а также легких ионов начиная с протонов, $\alpha$-частиц и т.д. Указанные возможности циклотрона ФТИ позволяют проводить на нем широкий спектр работ в области модифицирования различных структур, в том числе полупроводниковых, в области мембранных технологий и др., что в свою очередь требует эффективной работы ускорителя при ускорении различных ионов. Полное сечение перезарядки ионов на остаточном газе зависит от атомного номера и заряда иона, его энергии, состава и давления остаточного газа [3]. А поскольку характеристики и устройство циклотрона ФТИ значительно отличаются от большинства существующих ускорителей, представлялось интересным и полезным проведение исследований состава остаточного газа в ускорительном комплексе циклотрона ФТИ, чего ранее не делалось.

Вакуумная система ускорительного комплекса ФТИ схематически представлена на рис. 1. Она состоит из камеры циклотрона, ионопровода переменного сечения длиной $15 \mathrm{~m}$, вакуумных камер (отмечены цифрами 8 и 9) для облучения различных материалов, включая рулонные полимерные (камера 9), и группы откачных устройств (позиции 1-7). Вакуумная камера циклотрона представляет собой цельное латунное кольцо с внутренним диаметром $1120 \mathrm{~mm}$ и две крышки из АРМКО-железа, внутренние поверхности которых плакированы слоем меди. В камеру введены и закреплены на кольце PIG-источник, дуанты и электрод дефлектора канала вывода пучка. Перечисленные элементы уплотнены жгутом вакуумной резины. Ионопровод и камеры мишеней изготовлены из нержавеющей стали. На всей длине ионопровода расположены многочисленные вводы различных устройств, системы измерения тока и диагностики ускоренных пучков, различные диафрагмы, элементы систем формирования пучков и смотровые окна из оргстекла или толстого стекла, которые уплотнены преимущественно с помощью витона, а также вакуумной резины. Для визуальной диагностики используется несколько подвижных экранов с нанесенным люминесцирующим составом. Откачка системы осуществляется 


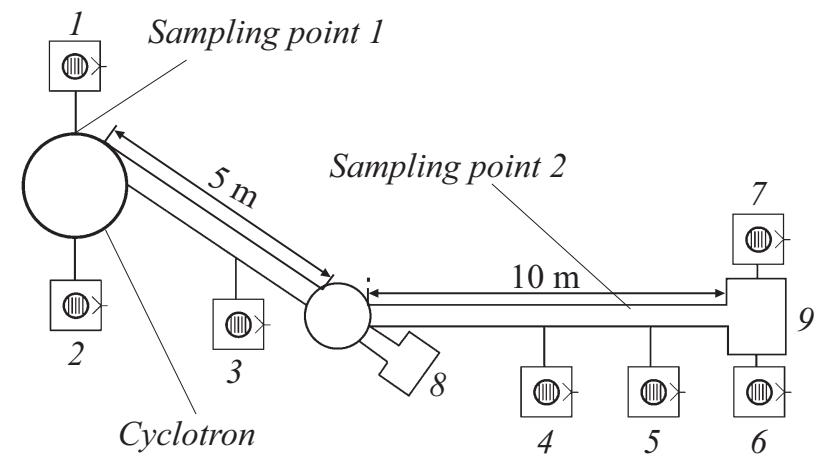

Рис. 1. Схема вакуумной системы циклотрона с ионопроводом. $1-7$ - турбомолекулярные насосы, $8,9-$ камеры для облучаемых материалов, точки 1 и 2 - места подсоединения масс-рефлектрона ФТ-200 к исследуемой системе.

турбомолекулярными насосами (позиции $1-7$ на рис. 1) с разными скоростями откачки от 500 до 2000 1/min. Система подачи рабочего газа в ионный источник выполнена из немагнитных медных трубок внутри ионного источника и нержавеющих трубок вне его.

Исследования состава остаточных газов проводились с помощью разработанного в ФТИ малогабаритного времяпролетного масс-спектрометра ФТ-200, который при длине масс-анализатора порядка $0.5 \mathrm{~m}$ обладает разрешающей способностью $~ 200$ на полувысоте массовой линии в области средних масс 20-50 u. Прибор имеет линейную траекторию движения ионов, т.е. ионный источник, отражатель и детектор расположены на одной прямой [4-6]. Обычно приборы такого типа называются масс-рефлектронами. В приборе используется источник ионов с электронным ударом, энергия ионизирующих электронов может изменяться от единиц до $130 \mathrm{eV}$ и выше, а энергия вылетающих ионов достигает $\sim 1200 \mathrm{eV}$, что определяется амплитудой выталкивающих импульсов, следующих с частотой $10 \mathrm{kHz}$.

Перед подключением масс-спектрометра к вакуумной системе циклотрона он был тщательно прогрет и обезгажен для того, чтобы уменьшить фоновые линии в спектре остаточного газа самого инструмента. На рис. 2, $a$ представлен масс-спектр прибора при остаточном давлении $<10^{-7}$ Torr, который вычитался из всех далее полученных спектров. На этом спектре и всех последующих интенсивность массовых пиков по оси ординат дана в произвольных единицах, а по оси абсцисс для однозарядных ионов отношение массы к заряду $m / z$ равно $m$. В приведенном спектре каждой массе соответствует свой тип ионов, за исключением неразрешенного триплета масс при $m=28 \mathrm{u}$, который содержит ионы ${ }^{14} \mathrm{~N}_{2}^{+}-{ }^{12} \mathrm{C}^{16} \mathrm{O}^{+}-{ }^{12} \mathrm{C}_{2}{ }^{1} \mathrm{H}_{4}^{+}$, для разделения которых необходима разрешающая способность порядка 5000 по основанию массовой линии.

Подключение масс-спектрометра к вакуумной системе циклотрона производилось в двух точках, отмеченных на рис. 1. Точка 1 расположена на кольце камеры циклотрона, в этом случае масс-рефлектрон подключался к циклотрону через насос (позиция 1 на рис. 1). Точка 2 подключения масс-рефлектрона находилась в ионопроводе вблизи камеры облучения крупноразмерных мишеней, в том числе пленочных материалов (позиция 9 на рис. 1). Масс-спектры регистрировались при различных режимах работы циклотронного комплекса. Все измерения проводились в вакуумной системе при открытых вакуумных шиберах и заслонках и всех работающих насосах. При достаточно длительной откачке системы, отсутствии магнитного поля, высокочастотного поля и напряжения на дефлекторе, а также при закрытой магистрали подачи рабочего газа в ионный источник, т.е. в „стационарном“ режиме откачки, давление остаточного газа в камере циклотрона, составляло $\sim 7 \cdot 10^{-7}$ Torr.

На рис. 2, $b$ приведен типичный масс-спектр остаточного газа в точке 1 при давлении в системе $\sim(5-7) \cdot 10^{-7}$ Torr, при этом давление в массрефлектроне составляло $\sim 5 \cdot 10^{-8}$ Torr, такой перепад давлений позволял регулировать напуск пробы в анализатор. Как видно из спектра, максимальный по амплитуде пик соответствует $m=18 \mathrm{u}$, т. е. ионам $\mathrm{H}_{2} \mathrm{O}^{+}$.

Обработка масс-спектра и расчет содержания компонент по площадям массовых пиков показывают, что вода составляет порядка 15-17 vol.\%, а пики органики, вероятно обусловленные парами вакуумного масла, начиная с $m=45-48$ u и до $m=96-99$ u дают от 70 до 82 vol.\% от суммы всех ионных токов, обусловленных молекулами остаточного газа в точке 1 вакуумной системы циклотрона. На рис. 2, с приведен масс-спектр остаточного газа также в точке 1 , но при напуске в ионный источник небольшого количества рабочего газа ${ }^{40} \mathrm{Ar}$, имитирующего штатную работу ионного источника, которое изменило давление в системе на $\sim 5 \cdot 10^{-8}$ Torr.

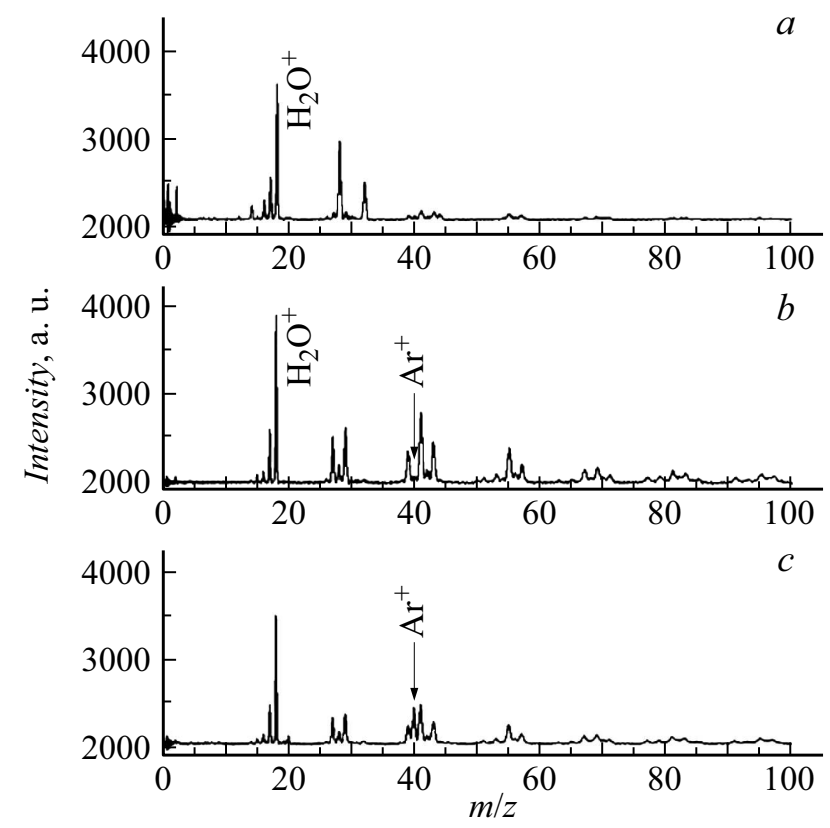

Рис. 2. Масс-спектры остаточного газа в масс-анализаторе ФТ-200 $(a)$, в точке 1 вакуумной камеры циклотрона $(b)$ и в точке 1 при напуске в камеру циклотрона малого количества Ar. 


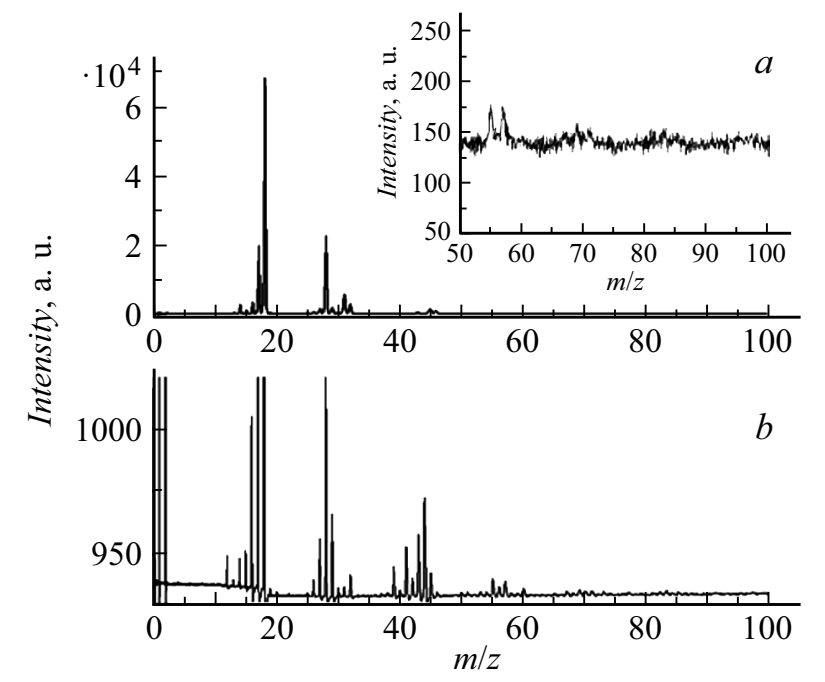

Pис. 3. $a-$ масс-спектр остаточного газа в точке 2 ионопровода в присутствии рулона лавсановой пленки. На вставке показан участок спектра паров масла в увеличенном масштабе по оси ординат. $b-$ масс-спектр паров масла, зарегистрированный при комнатной температуре.

На масс-спектре можно видеть пики ${ }^{40} \mathrm{Ar}^{+}$и ${ }^{40} \mathrm{Ar}^{++}$. Вклад ионного тока аргона в общий ток составил менее $10 \%$ от суммы токов.

Измерениям остаточного вакуума в точке 2 предшествовала продолжительная предварительная откачка системы, в результате которой устанавливалось равновесное, но неравномерное распределение давления остаточного газа. При этом в камеру мишеней был помещен рулон лавсановой пленки. В такой конфигурации давление остаточного газа на участке между насосами 4 и 5 (ближе к 4) составляло (7-9) $\cdot 10^{-6}$ Torr, а в камере $9-2.5 \cdot 10^{-4}$ Torr.

На рис. 3, $а$ представлен один из масс-спектров, полученных в точке 2 в присутствии рулона лавсановой пленки при напуске остаточного газа из ионопровода в массрефлектрон до давления $\sim 5 \cdot 10^{-7}$ Torr. Максимальный по амплитуде пик (примерно 68000 а.u.) соответствует ионам воды, он в 15 раз больше, чем в точке 1 при том же самом давлении пробы. Неразрешенный массовый пик при $m=28$ и также примерно в 10 раз превышает этот пик, измеренный в точке 1 . На вставке к рис. $3, a$ изображен фрагмент спектра от $m / z=50$ до $\mathrm{m} / z=100$ в увеличенном масштабе по оси ординат для удобства сравнения с рис. $3, b$, на котором представлен отдельно измеренный спектр паров вакуумного масла, используемого при эксплуатации циклотрона. Оценка содержания компонент по площадям соответствующих пиков в массспектрах показывает, что содержание воды в остаточном спектре в точке 2 составляет в разных экспериментах от 51 до 63 vol.\%, на неразрешенный пик при $m=28 \mathrm{u}$ приходится от 20 до 32 vol.\%, а площади массовых пиков при $m=19,31,45$ и $46 \mathrm{u}$, которых не было на спектрах, измеренных в точке 1 и которые мы не смогли точно идентифицировать, в сумме составляют от 8 до 11 vol.\%.
Возможно, это какие-то осколочные ионы вакуумного масла, которое традиционно в течение многих лет применялось в ионопроводе циклотрона ФТИ для смазки трущихся подвижных деталей, или осколочные ионы этанола $\mathrm{C}_{2} \mathrm{H}_{5} \mathrm{OH}$, которым протираются вакуумные детали при вскрытии камеры циклотрона или ионопровода. По крайней мере масс-спектр масла, показанный на рис. 3, $b$, содержит пики при $m=19,31,45,46 \mathrm{u}$, а также пики вплоть до $m=96-99$ u. К сожалению, в контрольном эксперименте, который проводился на другом массрефлектроне, масс-спектр следовых количеств этого вакуумного масла был зарегистрирован при давлении в анализаторе $\sim 10^{-8}$ Torr при комнатной температуре, поэтому амплитуды линий на высоких массах (выше $m=59$ u) оказались очень маленькими. В то же время попытка обнаружить какие-то вещества на лавсановой пленке из облучаемого рулона не дала результата, и полученный спектр дублировал спектр, представленный на рис. 2,a. По всей видимости, идентификация и установление происхождения пиков при $m=19,31$ и 45-46 u потребуют дальнейшего изучения.

Проведенные масс-спектрометрические исследования показали, что основными компонентами остаточного газа в камере циклотрона ФТИ и ионопроводе являются вода и пары масел, которые существенно влияют на эффективность вывода ускоренных пучков ионов, в первую очередь многозарядных, а также на точность электрометрических измерений в связи с перезарядкой ионов. Представляется полезным на первом этапе модернизации циклотрона ФТИ установить криогенную технику для вымораживания паров воды в самом циклотроне и, особенно, в ионопроводе, а также минимизировать давление паров масла за счет подбора оптимальных смазывающих материалов. Полученные результаты также будут использованы в дальнейших исследованиях перезарядки ускоренных многозарядных ионов. Эти работы необходимо проводить при постоянном массспектрометрическом контроле остаточных газов в вакуумной системе прибора.

\section{Конфликт интересов}

Авторы заявляют, что у них нет конфликта интересов.

\section{Список литературы}

[1] Гульбекян Г.Г., Иваненко А.И., Гикал Б.Н. // ЖТФ. 1984. T. 44. B. 7. C. $1288-1293$.

[2] Гикал Б.Н. Новое поколение циклотронов тяжелых ионов для прикладных исследований и промышленного применения. Докт. дис. Дубна: ОИЯИ, Лаборатория ядерных реакций им. Г.Н. Флерова, 2013. 257 с.

[3] Карамышев О.В., Карамышева Г.А., Скрипка Г.М. // Прикладная физика. 2011. № 6. С. 121-126.

[4] Мамырин Б.А., Шмикк Д.В. // ЖЭТФ. 1979. Т. 76. В. 5. C. $1500-1505$.

[5] Пилюгин И.И., Ждан В.Т., Козловский А.В. // Массспектрометрия. 2018. Т. 15. № 4. С. 274-276.

[6] Аруев Н.Н. // УФН. 2017. Т. 187. № 1. С. 1-19. 